\title{
Coupling Explicit Volume Diffusion with $\Sigma-Y$ model for LES of Airblast Atomisation
}

\author{
Bosen Wang*, M. J. Cleary, A. R. Masri \\ School of Aerospace, Mechanical and Mechatronic Engineering, The University of Sydney, \\ Sydney 2006, NSW, Australia \\ *Corresponding author email: bosen.wang@sydney.edu.au
}

\begin{abstract}
A novel volume of fluid (VoF) model based on an explicit volume diffusion (EVD) concept has been developed for interfacial flows. The EVD transport equations are derived by Favre volume averaging of VoF equations over a physically-defined volume length scale. This physical length scale is linked to the boundary layer thickness at the interface but is independent of the numerical grid size. A volume diffusivity and a related volume viscosity as functions of volume scale parameters have been derived to model the sub-volume flux and stress, respectively. A closure for volume averaged surface tension is also provided where a surface density of the physical volume is involved and needs to be closed. Here, a volume surface density transport equation is directly solved and this analogises to the one inherent to $\Sigma-Y$ model. The full EVD model (EVD coupled with $\Sigma-Y$ ) is validated for a laboratory airblast acetone spray jet. The EVD equations are discretised on the numerical grid. Keeping the volume length scale constant while reducing the numerical grid size, volume diffusion overwhelms the diminishing numerical diffusion leading to numerical convergence. The accuracy of the EVD method is evaluated by comparison to the available experimental data of liquid volume fraction.
\end{abstract}

\section{Keywords}

Explicit volume diffusion, volume fraction, surface density, surface tension force

\section{Introduction}

The interfacial flow including primary spray atomisation is featured by a sharp interface between two immiscible fluids. The numerical simulations of interfacial flows have evolved for decades with the improvements of high-performance computational resources and the development of numerical methods. Volume of fluid (VoF) [1], level set (LS) [2] and diffuse-interface (DI) [3] are three prominent methods. Since the VoF method is intrinsically mass conservative and its numerical solution is generally stable, we select it for further development. One main drawback of the general VoF method is the numerical diffusion due to the discretisation of VoF governing equations which leads to the continuously varying volume fraction values between 0 and 1 . Geometric VoF by sharpening and reconstructing surfaces [4] is required for the reduction of the intrinsic numerical diffusion. In spite of surface sharpening, direct/detailed numerical simulations (DNS) with highly refined grids are generally needed to minimise numerical diffusion and to resolve the key topological features of the interface [5], but the computational cost is enormous.

Large eddy simulations (LES) requiring much lower grid resolution for only resolving large eddy scales have the potential to vastly reduce the computational expense. However, accurate closures are required to close the sub-grid small scale fluctuations. Many LES of turbulent interfacial flows simply applied a conventional turbulence filter to the VoF transport equations and used standard turbulent viscosity and turbulent diffusivity models to close sub-grid stress and flux, respectively $[6,7]$. However, interfacial flows are conceptually different from single phase flows in several aspects such as: (i) the energy cascade and decay are not statistically universal across a sharp interface; (ii) the smallest length scale is unknown and approaches zero; (iii) not only turbulence, but also inhomogeneities induced by the dynamics of the interface lead to 
sub-grid fluctuations. Especially for interfacial flows in low Reynold number turbulent and laminar flows, the sub-grid turbulent diffusivity is not sufficient for modelling the sub-grid fluctuations induced by inhomogeneous phases due to the existence of the interface. Additionally, the intrinsic numerical diffusion of discretised VoF equations is amplified in LES for larger grid scales which is significant but has rarely been investigated. Our recent work [8] has demonstrated that rigorous numerical convergence cannot be achieved by this turbulence filtering VoF (TF-VoF) approach. A hybrid turbulence filtering and artificial compression method (TF-AC) was developed in [9] where an artificial compression term [10] is used to minimise numerical diffusion in regions near the continuous interface. Beyond these regions, the turbulent diffusivity model is alternatively used to describe the sub-grid dispersion of various liquid fragments. This TF-AC model was also evaluated in our recent work [8] and was demonstrated to be grid dependent leading to diverging results on refined grids.

Wang et al. [11] developed a new approach based on the basic VoF algorithm which is called explicit volume diffusion (EVD). This model can be applied to laminar and turbulent flows and is compatible with LES. An explicitly-defined physical volume length scale is introduced over which VoF transport equations are averaged. This volume averaging operation is conceptually different from turbulence filtering in that it attenuates the fluctuations induced by both interface dynamics and turbulence. Volume averaging effectively smears the interface relative to the volume length scale rather than the grid scale, and the unresolved interface dynamics need to be modelled. Unclosed sub-volume flux, sub-volume stress and volume averaged surface tension force terms appear in the EVD transport equations. The sub-volume flux is closed by a gradient diffusion model with a volume diffusivity coefficient. Similarly, a volume viscosity model augmented by an effective turbulent viscosity is derived to close the sub-volume stress. The closure for volume averaged surface tension force has been also derived where a volume surface density parameter is involved. The volume surface density was modelled based on fractal properties of wrinkled sub-volume interfaces as a first attempt [11]. The present study proposes to close it by a volume surface density transport equation which is analogous to the one inherent to the well-known $\Sigma-Y$ model $[6,12]$. For the EVD simulations, the physical volume averaged equations are discretised on the LES grid. When refining the grids, the explicit volume diffusion is still computed by the parameters taken from the constant physical volume. This physical defined volume diffusion is employed to account for a "diffusion" between the two immiscible fluids. Meanwhile, numerical diffusion is reduced on the small grid scales which will be overwhelmed by volume diffusion leading to numerical convergence. The accuracy of the EVD method is evaluated using an airblast atomising acetone spray jet, for which high quality measurements of volume fraction of liquid are available.

\section{The explicit volume diffusion (EVD) model}

The EVD model is obtained by averaging the VoF equations over physically-defined volumes $(V)$ with a characteristic length scale of $l_{V} \sim V^{1 / 3}$. Applying the Favre volume averaging operator to instantaneous VoF equations leads to [11]

$$
\begin{aligned}
& \frac{\partial \bar{\rho}}{\partial t}+\frac{\partial \bar{\rho} \widetilde{u_{i}}}{\partial x_{i}}=0 \\
& \frac{\partial \bar{\rho} \widetilde{u_{i}}}{\partial t}+\frac{\partial \bar{\rho} \widetilde{u_{i}} \widetilde{u_{j}}}{\partial x_{i}}=-\frac{\partial \bar{p}}{\partial x_{i}}+\frac{\partial}{\partial x_{i}}\left[\bar{\mu}\left(2 \widetilde{S_{i j}}-\frac{2}{3} \frac{\partial \widetilde{u_{k}}}{\partial x_{k}} \delta_{i j}\right)\right]+\overline{F_{s, i}}+\bar{\rho} g_{i}-\frac{\partial \tau_{i j}^{v a}}{\partial x_{i}}, \\
& \frac{\partial \bar{\rho} \widetilde{\alpha}}{\partial t}+\frac{\partial \bar{\rho} \widetilde{u_{i}} \widetilde{\alpha}}{\partial x_{i}}=-\frac{\partial J_{\alpha, i}^{v a}}{\partial x_{i}} .
\end{aligned}
$$

The volume averaged single fluid density and viscosity are calculated by $\bar{\rho}=\bar{\alpha} \rho^{l}+(1-\bar{\alpha}) \rho^{g}, \bar{\mu}=$ $\bar{\alpha} \mu^{l}+(1-\bar{\alpha}) \mu^{g}$, respectively. Here, the superscripts, $l$ and $g$, represent liquid and gas, respectively. The Favre volume averaged volume fraction is directly solved and the physically 
meaningful Reynolds volume averaged volume fraction can be obtained by using the relations for the conservation of liquid mass, $\bar{\alpha}=\bar{\rho} \widetilde{\alpha} / \rho_{l}$. Closures have been derived for the unclosed sub-volume stress, $\tau_{i j}^{v a}$, the volume averaged surface tension force, $\overline{F_{s, i}}$ in Eq. (2) and the subvolume flux, $J_{\alpha, i}^{v a}$ in Eq. (3) in [11]. As in [8], only brief introductions of these sub-volume models are presented in this paper. Using the bimodal probability function concept, the closure for the sub-volume flux can be modelled by

$$
J_{\alpha, i}^{v a}=\widetilde{\rho} \alpha^{\prime \prime} u_{i}^{\prime \prime}=\bar{\rho} \widetilde{\alpha}(1-\widetilde{\alpha})\left(\widetilde{u}_{i}^{l}-{\widetilde{u_{i}}}^{g}\right)=-\bar{\rho} D_{V} \frac{\partial \widetilde{\alpha}}{\partial x_{i}},
$$

where

$$
D_{V}=C_{\alpha u}\left|\frac{Q}{E}\right|^{3 / 2} l_{V}^{2} \sqrt{\widetilde{\alpha}(1-\widetilde{\alpha})} \sqrt{2 \widetilde{S_{i j} \widetilde{S_{i j}}}}
$$

is called the explicit volume diffusion coefficient. The parameters involved for computations of $D_{V}$ are directly taken from the constant physical volume. When refining the grid for solving the discretised volume averaged transport equations, $D_{V}$ does not change statistically which accounts for a physical "diffusion" between two immiscible fluids. This volume diffusion overwhelms the decreasing numerical diffusion leading to numerical convergence. The difference between the mean velocities of liquid and gas, ${\widetilde{u_{i}}}^{l}-{\widetilde{u_{i}}}^{g}$, is called the mean drift velocity that essentially leads to the "diffusion" between the two phases. The coherent function, $|Q / E|$, characterises the relative magnitudes of vorticity and strain [13]. The second invariant of the velocity gradient tensor, $Q$, the magnitude of a velocity gradient tensor, $E$ and the vorticity tensor, $\widetilde{W_{i j}}$ are given by

$$
Q=\frac{1}{2}\left(\widetilde{W_{i j}} \widetilde{W_{i j}}-\widetilde{S_{i j}} \widetilde{S_{i j}}\right), E=\frac{1}{2}\left(\widetilde{W_{i j}} \widetilde{W_{i j}}+\widetilde{S_{i j}} \widetilde{S_{i j}}\right), \widetilde{W_{i j}}=\frac{1}{2}\left(\frac{\partial \widetilde{u_{i}}}{\partial x_{j}}-\frac{\partial \widetilde{u_{j}}}{\partial x_{i}}\right),
$$

respectively. High values of $|Q / E|$ occur in homogeneous or strong shear turbulence and leading to high values of $D_{V}$ and strong dispersion of the interface. Low turbulence and laminar conditions require low $|Q / E|$. An interesting limit occurs where the interface curvature approaches zero with $|Q / E| \rightarrow 0$ and as a result $D_{V} \rightarrow 0$. This limiting behaviour ensures that there is not premature dispersion of the liquid into the gas and this is particularly important along continuous interfaces prior to the appearance of curvature generating instabilities. This ensures $D_{V}$ based on interfacial physics which increases smoothly as the interface curvature increases. Another limit occurs away from the interface where the term $\sqrt{\widetilde{\alpha}(1-\widetilde{\alpha})}$ in Eq. (5) goes to zero and therefore $D_{V}=0$. This is correct because $\alpha$ is a constant (either zero or one) in the interior of the pure fluid streams and therefore sub-volume flux cannot exist. A priori analysis against resolved simulations in Wang et al. [11] suggests that the modelling constant $C_{\alpha u}=0.25$.

The closure for sub-volume stress is approximated by

$$
\tau_{i j}^{v a}=-2 \bar{\rho}\left(\nu_{V}+\nu_{t}^{e f f}\right) \widetilde{S_{i j}}
$$

An explicit volume viscosity coefficient is introduced as $\nu_{V}=D_{V} S c_{V}$, where $S c_{V}=3$ is an explicit volume Schmidt number whose value was also determined by a priori analysis [11]. As indicated by the closure of $D_{V}$, in the interior of the pure liquid and gas phases and at any place where $|Q / E| \rightarrow 0, D_{V}=0$ thus $\nu_{V}=0$, however, sub-volume turbulent velocity fluctuations can exist at those locations and $\tau_{i j}^{v a}$ is reverted to the sub-filter turbulent stress. This is characterised by an effective turbulent viscosity, $\nu_{t}^{\text {eff }}=[1-4 \widetilde{\alpha}(1-\widetilde{\alpha})] \nu_{t}$, where $\nu_{t}$ can be modelled by the

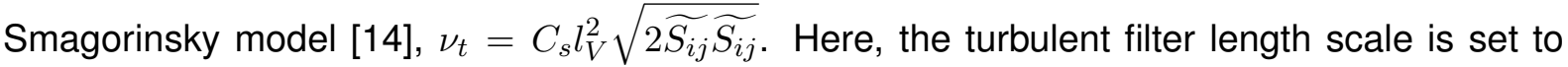
be the same as the volume length scale. The interface sensor $[1-4 \widetilde{\alpha}(1-\widetilde{\alpha})]$ is a smooth blending function inspired by the flame sensor function employed in the artificially thickened flame model to account for the effects of turbulence on premixed flame interfaces. The low sensitivities of $C_{\alpha u}$ and $S c_{V}$ have been demonstrated in [11]. 
The continuum surface tension model [15] is widely used for LES and DNS of spray atomisation $[5,6,7]$. However, modelling of sub-grid fluctuations of the surface tension force is a longstanding challenge which is usually neglected in most of LES studies [6, 7]. We derive a model for volume average surface tension where a volume surface density, $\bar{\Sigma}$, is involved [11],

$$
\overline{F_{s, i}} \cong \sigma \overline{\kappa n_{i}} \bar{\Sigma} \text {. }
$$

Here, $\bar{\kappa}=-\nabla \cdot \bar{n}$ denotes the curvature of the volume averaged interface with the normal direction $\overline{\boldsymbol{n}}=\nabla \bar{\alpha} /|\nabla \bar{\alpha}|$. A volume surface density, $\bar{\Sigma}$, is generally defined as the physical volume divided by the sub-volume total area of interfaces which is modelled based on fractal properties of wrinkled sub-volume interfaces as a first attempt [8,11]. A new approach to close $\bar{\Sigma}$ is presented as follows.

\section{Coupling EVD with $\Sigma-Y$ model}

Instead of using the fractal wrinkled sub-volume interface model, the volume surface density in Eq. (8) can be solved by a transport equation similar to the one inherent to $\Sigma-Y$ model. To be consistent with the Favre volume averaged volume fraction, $\widetilde{\Omega}$ is defined as the surface area based on the mass in the physical volume. Their relationship is given by $\bar{\Sigma}=\bar{\rho} \widetilde{\Omega}$. The transport equation for $\widetilde{\Omega}$ can be written as [12]

$$
\frac{\partial \bar{\rho} \widetilde{\Omega}}{\partial t}+\frac{\partial \bar{\rho} \widetilde{\Omega}\left\langle u_{i}\right\rangle_{s}}{\partial x_{i}}=\overline{S_{\Omega}},
$$

where $\left\langle u_{i}\right\rangle_{s}$ represents the surface averaged velocity, $\overline{S_{\Omega}}$ is the source term. The surface averaged velocity can be decomposed into the bulk velocity $\widetilde{u_{i}}$ and their deviation $\left\langle u_{i}\right\rangle_{s}-\widetilde{u_{i}}$ leading to

$$
\frac{\partial \bar{\rho} \widetilde{\Omega}}{\partial t}+\frac{\partial \bar{\rho} \widetilde{\Omega} \widetilde{u_{i}}}{\partial x_{i}}=-\frac{\partial}{\partial x_{i}}\left[\bar{\rho} \widetilde{\Omega}\left(\left\langle u_{i}\right\rangle_{s}-\widetilde{u_{i}}\right)\right]+\overline{S_{\Omega}} .
$$

The term $\left\langle u_{i}\right\rangle_{s}-\widetilde{u_{i}}$ can be considered as the volume diffusive velocity of surface density. On the heavy fluid side, the boundary layer thickness is very small [16] such that the average interface velocity can be approximated as the average bulk velocity of the heavy fluid in the volume, i.e. $\widetilde{u}_{i}^{l}=\left\langle u_{i}\right\rangle_{s}$, and thus the sub-volume diffusive velocity of surface density becomes ${\widetilde{u_{i}}}^{l}-\widetilde{u_{i}}$. Substituting the relationship $\widetilde{u_{i}}=\widetilde{\alpha}{\widetilde{u_{i}}}^{l}+(1-\widetilde{\alpha}) \widetilde{u}_{i}^{g}$ into Eq. (4) leads to the sub-volume diffusive flux of volume fraction being $J_{\alpha, i}^{v a}=\bar{\rho} \widetilde{\alpha}\left(\widetilde{u}_{i}^{l}-\widetilde{u_{i}}\right)$. Therefore, the sub-volume diffusivity velocity for volume fraction is also $\widetilde{u}_{i}^{l}-\widetilde{u_{i}}$ which is the same as the approximated diffusive velocity of surface density. Therefore, the volume diffusion coefficient can also be employed for modelling the sub-volume dispersion of surface density,

$$
\frac{\partial \bar{\rho} \widetilde{\Omega}}{\partial t}+\frac{\partial \bar{\rho} \widetilde{\Omega} \widetilde{u_{i}}}{\partial x_{i}}=\frac{\partial}{\partial x_{i}}\left[\bar{\rho} D_{V} \frac{\partial \widetilde{\Omega}}{\partial x_{i}}\right]+\overline{S_{\Omega}} .
$$

The term, $\widetilde{\Omega}$, is decomposed into a minimum part, $\widetilde{\Omega}_{\text {min }}$, which implies the presence of an interface simply due to the coexistence of liquid and gas phases, and an additional sub-volume dynamic part, $\widetilde{\Omega_{d}}$, due to the wrinkling and stretching induced by sub-volume velocity fluctuations, and the coalescence and breakup of liquid structure interactions. Here, $\widetilde{\Omega}_{\min }$ is approximated by a algebraic relationship, $\widetilde{\Omega}_{\text {min }}=C_{\Sigma_{\min }} \sqrt{\bar{\alpha}(1-\bar{\alpha})} / l_{V} / \bar{\rho}$, where the modelling constant is suggested as $C_{\Sigma_{\min }}=2.4$ [6]. The transport equation can be solved to obtain $\widetilde{\Omega_{d}}$,

$$
\frac{\partial \bar{\rho} \widetilde{\Omega_{d}}}{\partial t}+\frac{\partial \bar{\rho} \widetilde{\Omega_{d}} \widetilde{u_{i}}}{\partial x_{i}}=\frac{\partial}{\partial x_{i}}\left[\bar{\rho} D_{V} \frac{\partial \widetilde{\Omega_{d}}}{\partial x_{i}}\right]+\overline{S_{\Omega_{d}}} \text {. }
$$

The source term $\overline{S_{\Omega_{d}}}$ is approximated by a similar formula taken from the source term for the $\Sigma-Y$ model, $\overline{S_{\Omega_{d}}}=C_{S_{\Sigma}} \bar{\rho} \widetilde{\Omega}\left(1-\widetilde{\Omega} / \Omega^{*}\right) / \tau_{V}=C_{S_{\Sigma}} \bar{\Sigma}\left(1-\bar{\Sigma} / \Sigma^{*}\right) / \tau_{V}$, where the modelling constant $C_{S_{\Sigma}}$ was proposed as 0.4 [17]. The characteristic volume time scale reads as $\tau_{V}=$ 
$1 / \sqrt{\widetilde{s_{i j} \widetilde{s_{i j}}}}$. In order to maintain model consistency, the equilibrium surface density is also proposed to contain a sub-grid scale contribution and the minimum surface density, $\Sigma^{*}=\bar{\rho} \widetilde{\Omega}_{m i n}+$ $2 \bar{\alpha}(1-\bar{\alpha})\left(\rho_{l}+\rho_{g}\right) \widetilde{k} /\left(\sigma W e^{*}\right)$, where $W e^{*}$ is a critical Weber number and is suggested as 1.63 [17]. The sub-volume kinetic energy $\widetilde{k}$ is given by $\widetilde{k}=3\left|u_{V}^{\prime \prime}\right|^{2} / 2=3\left(C_{S L} l_{V} \sqrt{2 \widetilde{S_{i j}} \widetilde{S_{i j}}}\right)^{2} / 2$, where $\left|u_{V}^{\prime \prime}\right|$ represents the characteristic sub-volume velocity fluctuation induced by the drift velocity and turbulent velocity fluctuations. The modelling constant, $C_{S L}=0.66$, is proposed based an a priori analysis against resolved flow simulations [11]. It is evident that the source term, $\overline{S_{\Omega_{d}}}$, for volume surface density is a non-linear term. Computing $\overline{S_{\Omega_{d}}}$ based on the constant volume scale independent of the grid scale can accelerate the numerical convergence.

The explicit volume length scale, $l_{V}$, is supposed to be linked to a physical dimension of the interfacial flow. Based on the investigation of the effect of the magnitude of $l_{V}[11], l_{V}$ was suggest to be no larger than the boundary layer thickness on the gas side of the interface for which some empirical approximations are available [16]. Additionally, numerical convergence is achieved when $l_{V}$ is several times larger than the LES grid size, $\Delta$. In combination, these two requirements ensure that smearing of the boundary layer due to volume averaging is not excessive and that the explicit volume diffusion remains sufficiently large to overwhelm numerical diffusion.

\section{Numerical implementation}

The EVD model has been implemented in a new solver called evdFoam that is incorporated in OpenFOAM. The volume averaged transport equations are solved on the LES mesh giving grid values of the properties, e.g. $\bar{\rho}^{\Delta}, \widetilde{\alpha}^{\Delta}, \widetilde{\Omega}^{\Delta}$ and $\widetilde{u}_{i}{ }^{\Delta}$. A second mesh of length scale $l_{V}>\Delta$ is used for calculating the explicit volume diffusion coefficient in the interface region. This requires integrating each of the quantities in Eq. (5) over all LES cells inside each explicit volume. For example, the integrated volume averaged fields are given by $\widetilde{\alpha}^{V}=\int_{V} \bar{\rho}^{\Delta} \widetilde{\alpha}^{\Delta} d V / \bar{\rho}$, $\widetilde{u}_{i}^{V}=\int_{V} \bar{\rho}^{\Delta}{\widetilde{u_{i}}}^{\Delta} d V / \bar{\rho}$. Once $D_{V}^{V}$ is known, the values at the LES cells, $D_{V}^{\Delta}$, that are needed to solve the transport equations are obtained by linear interpolation. In the interior of each pure fluid, it is convenient and logical to make the volume and grid scales equal where turbulent viscosity is modelled to close the sub-grid stress. The integrated volume averaged surface density is also obtained and this is taken as input to Eq. (8) to close the volume averaged surface tension force, ${\overline{F_{s} i}}^{V}$. The magnitude of the force at each LES cell, ${\overline{F_{s}}}^{\Delta}$, is obtained by linear interpolation from $\bar{F}_{s}{ }^{V}$ on the explicit volume mesh. To better capture the interface dynamics, the direction of the force is given directly from the grid resolved interface normal direction which reads $\bar{n}^{\Delta}=\nabla \bar{\alpha}^{\Delta} /\left|\nabla \bar{\alpha}^{\Delta}\right|$.

The standard numerical schemes in OpenFOAM are employed for evdFoam $[10,8,11]$. It should be noted that the convection of volume fraction is solved by using a multidimensional universal limiter for explicit solution (MULES) algorithm [18]. MULES can guarantee the boundedness of volume fraction fields and numerical stability for immiscible interfaces with large liquid/gas density ratios. In the test cases investigated here, the maximum interface Courant number is $C o_{i}=0.25$ but since the numerical method integrates the volume fraction over four sub-steps such that it is effectively 0.0625 . The maximum flow Courant number is $C o_{f}=0.75$.

\section{Results and discussion}

A laboratory turbulent non-reacting acetone spray jet produced by airblast atomisation is selected as the test case for validating the full EVD approach (EVD coupling with $\Sigma-Y$ ). High quality measurements of volume fraction of liquid are obtained by using the two-angle backlit imaging technique $[8,19]$. The N-AS2 case is selected where the liquid acetone is injected from a needle with diameter $d_{j}=686 \mu \mathrm{m}$ and the turbulent air stream is injected from a flush concentric airblast tube with diameter $D=10 \mathrm{~mm}$. The densities and kinematic viscosities 
of air and liquid acetone are well-known and their respective values are $\rho_{g}=1.178 \mathrm{~kg} / \mathrm{m}^{3}$, $\rho_{l}=784.5 \mathrm{~kg} / \mathrm{m}^{3}, \nu_{g}=1.567 \mathrm{e}-5 \mathrm{~m}^{2} / \mathrm{s}$ and $\nu_{l}=3.938 \mathrm{e}-7 \mathrm{~m}^{2} / \mathrm{s}$. The inflow mean velocity, turbulent intensity, Reynolds number and the jet Weber number are shown in Table 1. The jet Weber number based on the interfacial drift velocity is 35 and is calculated by $W e=\rho_{g}\left(U_{g}-U_{l}\right)^{2} d_{j} / \sigma$.

Table 1. Parameter setup for the experimental airblast acetone spray, N-AS2

\begin{tabular}{c|ccccccc}
\hline Case & $U_{g}(\mathrm{~m} / \mathrm{s})$ & $\mathrm{U}_{l}(\mathrm{~m} / \mathrm{s})$ & $U_{g}^{\prime} / U_{g}$ & $U_{l}^{\prime} / U_{l}$ & $R e_{g}$ & $R e_{l}$ & $W e$ \\
\hline N-AS2 & 36 & 4.3 & 0.11 & 0.0525 & 21398 & 7491 & 35 \\
\hline
\end{tabular}

A three-dimensional cylindrical domain is created for computations with an axial length of $L_{x}=$ $50 \mathrm{~mm}$ and a diameter of $D_{i n}=5 \mathrm{~mm}$. The inflow turbulent perturbations for both air and liquid streams are generated by a digital filter [20]. The integral length scale of the airblast and the liquid jet are set to be $1 / 8$ of $D$ and $d_{j}$, respectively. Here, $D_{i n}$ is half the experimental airblast diameter, $D$, for the reduction of computational cost meanwhile it is sufficient to cover the spray primary breakup region without undue effects from the numerical boundary conditions. The characteristic boundary thickness on the gas side of the interface [16] is approximated as $\zeta=198 \mu \mathrm{m}$. The explicit volume length scale in the interface region is $l_{V}=190 \mu \mathrm{m}$ which is just smaller than $\zeta$. Three different grid resolutions containing 1.1M, 2.3M and $4.5 \mathrm{M}$ ( $\mathrm{M}$ denotes million) cells are used to discretise the EVD transport equations and the reader is referred to $[8,11]$ for a detailed description of the mesh scheme. The corresponding ratios of grid to explicit length ratios are $\Delta / l_{V}=0.26,0.2$ and 0.16 , respectively.

Figure 1 shows instantaneous volume fraction and surface density fields at the centre plane of the computational domain. It can be visualised from the $\bar{\alpha}^{\Delta}$ field (top) that the interface instability evolves and the amplitude of the wave amplitudes grow. The liquid core gradually departs from the centre line and tends to be pinched off at about $x=0.0125 \mathrm{~m}$. At about $x=0.015 \mathrm{~m}$ large irregular objects of liquid are formed which subsequently break up into various smaller fragments. The volume integrated volume fraction field is presented $\left(\bar{\alpha}^{V}\right.$, middle). Compared with $\bar{\alpha}^{\Delta}$, only the interface regions are smoothed due to volume averaging over a volume scale of the characteristic boundary layer thickness but the key features of different types of fragments remain unchanged. Before the breakup of the liquid jet, the surface density (bottom) first rises along the continuum interface due to interface wrinkling induced by large sub-volume velocity fluctuations. Subsequently, it decreases to a moderate magnitude. This indicates the interface wave length grows larger and the number of wrinkles in the finite volume tends to reduce. As the jet starts to break, the surface density increases in the centre line. Further downstream, intermittency can be observed due to discrete interfaces of different fragments which also disperse radially. This leads to the decay of surface density along with volume fraction.

Figure 2 shows the mean and rms of volume fraction and surface density in the axial and radial directions. The experimental measurements of volume fraction in the axial direction are available [19] that are taken to compare with the statistics of the simulations. The experimental error bars relative to the mean values are used to validate the rms of volume fraction. The mean and rms of volume fraction profiles in both axial and radial directions (at $x / D_{\text {in }}=3$ and 6) reach convergence by refining grids. Especially, the $2.3 \mathrm{M}$ and $4.5 \mathrm{M}$ lines roughly overlap in spite of slight deviation between $2.5<x / D_{\text {in }}<3.5$. Except for the discrepancy at $r / D_{\text {in }}=3$ for the rms profiles, the surface density also exhibit acceptable grid convergence for $2.3 \mathrm{M}$ and $4.5 \mathrm{M}$. Overall, the improvement of the model for $\overline{F_{s, i}}$ does not affect the good grid convergence as shown in [11] which is superior to the methods proposed in [6,9] as demonstrated in [8]. A slightly earlier breakup location is predicted by the converged numerical results. Subsequent underestimation of the mean volume fraction in the axial direction can be observed albeit still within the experimental error margin. The position of the highest rms is well predicted at about $x / D_{\text {in }}=3$ but the magnitude of the peak value is overpredicted. Upstream of this peak value, the predictions of rms are generally good, however, underpedictions are observed in the downstream region. Compared with the experimental data, the key inaccuracy of the modelling results lies 


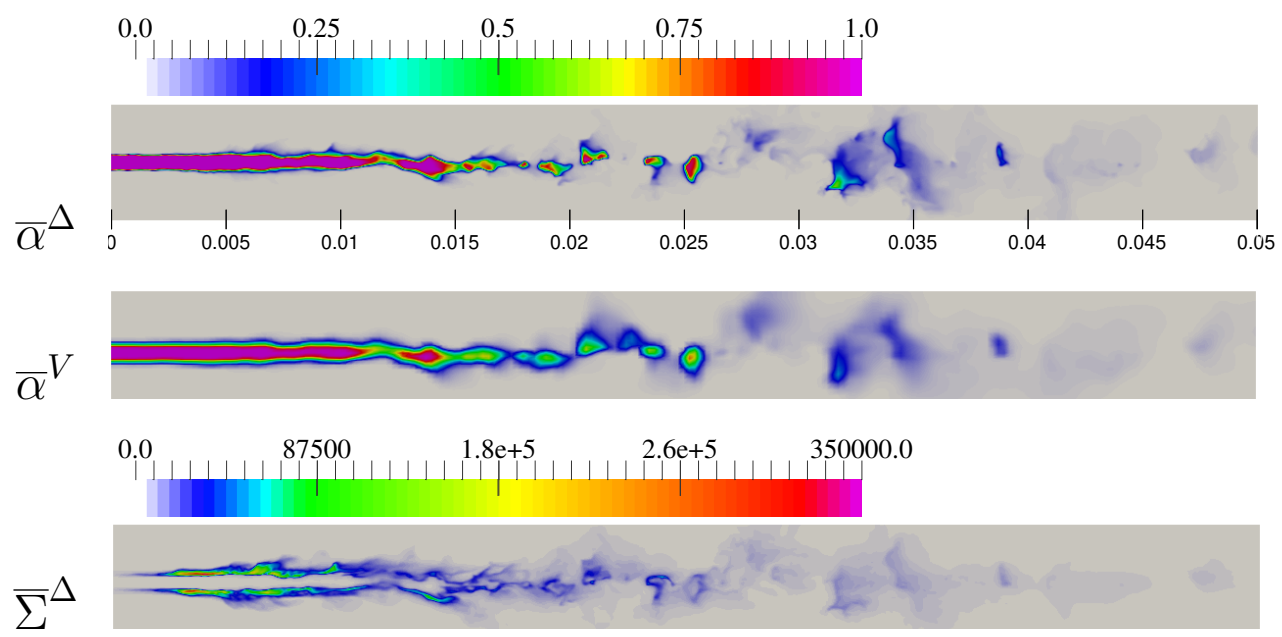

Figure 1. Comparisons of instantaneous volume fraction fields on the 4.5M LES grid $\left(\bar{\alpha}^{\Delta}\right)$ and on the volume mesh $\left(\bar{\alpha}^{V}\right)$, and the surface density fields on the LES grid $\left(\bar{\Sigma}^{\Delta}\right)$. The $x$-axis is added to the bottom of the image of $\bar{\alpha}^{\Delta}$ with the unit of meter.

in the overpredictions of the decay of the jet. This is likely to be improved by reducing the magnitude of volume averaged surface tension force [8] which can be simply realised by reducing the surface density source term. Thus, a smaller value of $C_{S_{\Sigma}}$ in the source term, $\overline{S_{\Omega_{d}}}$, or a larger critical Weber number, $W e^{*}$, can be tuned for further validation.
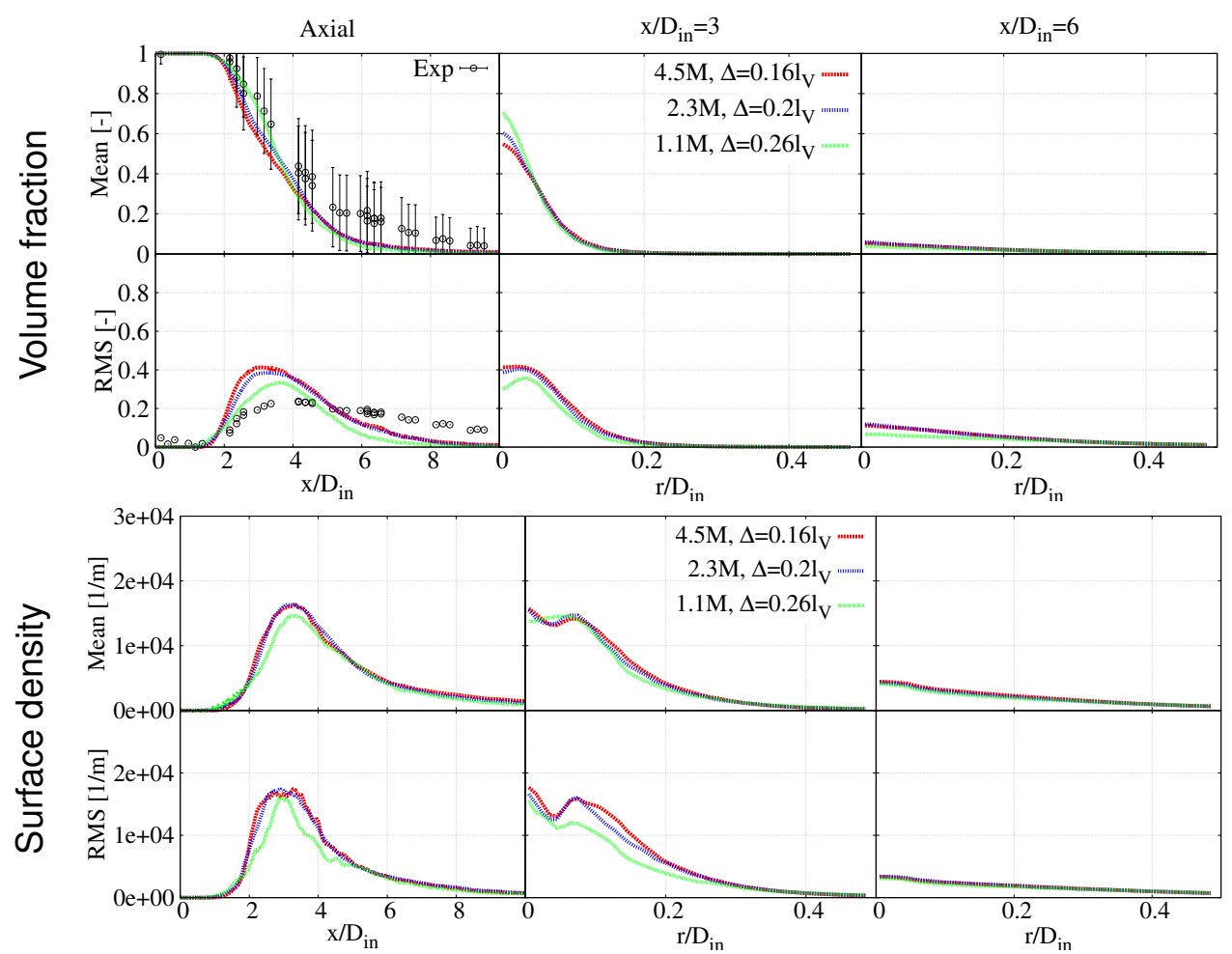

Figure 2. Axial and radial profiles of mean and rms of volume fraction and surface density. Radial profiles are shown at two axial stations. Experimental data [19] includes error bars.

\section{Conclusions}

A novel explicit volume diffusion (EVD) model is presented based on averaging the VoF equations over explicitly defined physical volumes [11]. The explicit volume scale is linked to the boundary layer thickness on the gas side of the liquid-air interface but is independent of the 
grid scale. Volume averaging leads to unclosed sub-volume flux and stress which are modelled by a physically derived explicit volume diffusion coefficient and a related explicit volume viscosity augmented by an effective turbulent viscosity, respectively. Another unclosed volume averaged surface tension force was derived to be correlated with a volume surface density that was modelled based on fractal properties of wrinkled sub-volume interfaces [11]. Alternatively, the volume surface density is proposed to be closed by solving its transport equation that can be analogised to the one inherent to $\Sigma-Y$ model in the present study. A laboratory airblast acetone spray jet is selected as the test case. The numerical convergence of this full EVD model is demonstrated by refining grids while keeping the physical volumes constant, so that the explicit volume diffusion does not essentially vary and overwhelms the diminishing numerical diffusion leading to numerical convergence of both mean and rms profiles of volume fraction and surface density. The converged results are compared with the experimental data of liquid volume fraction to access the model accuracy. A slightly earlier breakup location is predicted. Subsequent underestimation of the mean volume fraction in the axial direction can be observed albeit still within the experimental error margin. To improve the accuracy of computations, the sensitivity of parameters involved in the surface density source term is suggested to be tested. The full EVD model will be further validated against the high Weber number jet with $W e=72$ [19].

\section{Acknowledgements}

The financial support by the Australian Research Council and the HPC facilities at the University of Sydney are acknowledged. The authors also thank Dr Giovanni Tretola (University of Brighton) and Dr Salvador Navarro-Martinez (Imperial College) for discussions and clarifications of the $\Sigma-Y$ model.

\section{References}

[1] Hirt, C. W., Nichols, B. D., 1981, J. Comput. Phys., 39 (1), pp. 201-225.

[2] Sussman, M., Smereka, P., Osher, S., 1994, J. Comput. Phys., 114 (1), pp. 146-159.

[3] Anderson, D. M., McFadden, G. B., Wheeler, A. A., 1998, Annu. Rev. Fluid Mech., 30 (1), pp. 139-165.

[4] Roenby, J., Bredmose, H., Jasak, H., 2016, Royal Society Open Science, 3 (11), p. 160405.

[5] Hasslberger, J., Ketterl, S., Klein, M., Chakraborty, N., 2019, J. Fluid Mech., 859, pp. 819838.

[6] Chesnel, J., Reveillon, J., Menard, T., Demoulin, F. X., 2011, At. Sprays, 21 (9).

[7] Navarro-Martinez, S., 2014, International Journal of Multiphase Flow, 63, pp. 11-22.

[8] Abbas, F., Wang, B., Cleary, M. J., Masri, A. R., 2021, Phys. Fluids, 33 (4), pp. 042119.

[9] Anez, J., Ahmed, A., Hecht, N., Duret, B., Reveillon, J., Demoulin, F. X. 2019, Int. J. Multiph. Flow, 113, pp. 325-342.

[10] Weller, H. G., 2008, OpenCFD Ltd., Report TR/HGW, 4, p. 35.

[11] Wang, B., Cleary, M. J., Masri, A. R., 2021, Phys. Fluids, 33 (6), pp. 062111.

[12] Lebas, R., Menard, T., Beau, P. A., Berlemont, A., Demoulin, F. X., 2009, Int. J. Multiph. Flow, 35 (3), pp. 247-260.

[13] Kobayashi, H., 2005, Phys. Fluids, 17 (4), pp. 045104.

[14] Smagorinsky, J., 1963, Monthly weather review, 91 (3), pp. 99-164.

[15] Brackbill, J. U., Kothe, D. B., Zemach, C., 1992, J. Comput. Phys., 100 (2), pp. 335-354.

[16] Marmottant, P., Villermaux, E., 2004, J. Fluid Mech., 498, pp. 73.

[17] Duret, B., Reveillon, J., Menard, T., Demoulin, F. X., 2013, Int. J. Multiph. Flow, 55, pp. 130-137.

[18] Zalesak, S. T., 1979, J. Comput. Phys., 31 (3), pp. 335-362.

[19] Singh, G., Kourmatzis, A., Masri, A. R., 2020, Exp. Therm. Fluid Sci. , 115, pp. 110102.

[20] Klein, M., Sadiki, A. and Janicka, J., 2003, J. Comput. Phys., 186 (2), pp. 652-665. 